\title{
Wholly Owned Offshore Subsidiaries for IT Development: A Program of Research ${ }^{\mathrm{ii}}$
}

\author{
Roberto Evaristo \\ University of Illinois \\ at Chicago \\ evaristo@uic.edu
}

\author{
Jorge L. Nicolas \\ Audy \\ PUCRS, Brazil \\ audy@inf.pucrs.br
}

\author{
Rafael Prikladnicki \\ PUCRS, Brazil \\ rafael@inf.pucrs.br
}

\author{
Jairo Avritchir \\ jairo_avritchir@uol.com.br
}

\begin{abstract}
Information Technology (IT) offshore outsourcing is a non-reversible trend due to its cost implications, and as a result, target of much inquiry in different areas of knowledge. We propose to focus research efforts on a smaller niche within that larger set of questions: the wholly owned subsidiary (WOS) created with the purpose of IT development. In particular, our first step will be to develop a fully testable maturity model for such endeavors in the context of distributed IT projects. The proposed maturity model will not only identify best practices along difference maturity stages but also help to benchmark across similar organizations. Our preliminary research suggests that there is a consistent sequence of phases that this type of IT offshore sourcing follows.
\end{abstract}

\section{Introduction}

Software has become a vital component of almost every business. Success increasingly depends on using software as a tool for competitive advantage [5]. More than a decade ago, many organizations began to experiment with remotely located software development facilities (also called Distributed Software Development DSD) seeking lower costs and access to skilled resources. Economic forces are relentlessly turning national markets into global markets and spawning new forms of competition and cooperation reaching across national boundaries [1].

This change is having a profound impact not only on marketing and distribution but also on the way products are conceived, designed, constructed, tested, and delivered to customers. For these reasons, DSD has attracted a large research effort in software engineering $[1,3,8,14]$. The search for such competitive advantage forces organizations to search for external solutions in other countries. The two main options include offshore outsourcing (contracting services with an external organization located in another country) as well as offshore insourcing (contracting with a wholly owned subsidiary also located in another country). The first has become fairly common, but difficulties abound in trying to develop a relationship with an unknown foreign partner that is time and geographically distant. Such issues have led select organizations to create their own software development centers in countries like India, Russia, Brazil, Ireland, etc. Although some of the key difficulties such as cultural differences, trust creation/maintenance and coordination across time and space do remain, a crucial variable is removed: the concern with partner responsiveness, accountability, behavior under pressure and other contract-related issues.

That allows us to concentrate on the true task at hand of understanding the relationship across subsidiaries without the artificial veil caused by contract related behaviors. However, this bypassing brings into relief a whole different set of issues. In particular, what is a "new" issue for an organization entering such arrangement is in fact something already been dealt - in a variety of ways - by organizations that have started earlier. The wholly owned concept affects not only how cultural differences and closely linked issues such as trust development and maintenance are managed across the units, but also how distributed coordination, task allocation, strategic application choices and other equally important concerns are perceived and acted upon. Ultimately, one of the main purposes of this program of research is to shed light in this type of relationship by developing a maturity model for such endeavor.

In the next section we will develop a theoretical model to guide our research, followed by a detailed description of the research methodology planned, as well as variable selection. In the section 4 we will discuss some of the expected results and then develop conclusions.

\section{Theoretical Model}

\subsection{Distributed Software Development}

In the last decade large investments have enable the move from local to global markets in the process of creating new competition and collaboration forms [5]. In the same period of time, the global software market has undergone several crises: Not only a large number of project failures have plagued the industry, but also the increasing demand for new systems has been strongly affected by scarcity in appropriate competences. In such environment, DSD provides a feasible alternative. Several factors have accelerated this trend [5]:

\footnotetext{
${ }^{1}$ An earlier version has been accepted for publication at AMCIS 2004, New York, NY.
} 
- The need to have a global resource pool to successfully and cost-competitively employ resources, wherever located;

- The business market proximity advantages, including knowledge of customers and local conditions;

- The quick formation of virtual corporations and virtual teams to exploit market opportunities;

- Pressure to improve time-to-market by using time zone differences in "round-the-clock" development;

Organizations search for competitive advantage in terms of cost, quality and flexibility in software development, looking for productivity increases as well as risk dilution [14]. Many times the search for these competitive advantages forces organizations to search for external solutions in other countries (offshore sourcing). The key existing challenges are [5]:

- Strategic issues: the decision whether a particular project can be developed by globally dispersed teams - and where it can be better developed, as well as how to divide it across sites - is difficult. Some analysis considering the risk and benefit of projects dispersion can be necessary. Solutions are constrained by the resources available at the sites, their levels of expertise in various technologies, infrastructure, etc;

- Cultural issues: offshore sourcing requires close cooperation of individuals with different cultural backgrounds. Cultures differ on many critical dimensions, such as national, ethnic, organizational, professional, technical, and team culture. While many people find such differences enriching, they can also lead to serious misunderstanding, especially among people who do not know each other well;

- Technical issues: when teams are working across sites, the lack of synchronization can be particularly critical. We need to assure a common defined milestones and clear entry and exit criteria for all tasks. The overhead of control (the process of adhering to goals, policies or standards) and coordination (the act of integrating each task and organizational unit so that it contributes to the overall objective) associated with any software projects is considerable;

- Knowledge management: without effective information and knowledge-sharing mechanisms, it is difficult to exploit the benefits of offshore sourcing. Poor documentation can cause ineffective collaborative development.

Offshore outsourcing provides a feasible alternative to implement DSD and concurrently take advantage of the lower cost inherent in other markets. In the next section, we will discuss further the concepts behind offshore sourcing, and immediately afterwards some of the shortcomings in traditional offshore outsourcing and how the implementation of a wholly owned subsidiary arrangement is able to get around many of them.

\subsection{Offshore Sourcing}

Offshore sourcing of IT work is increasingly occupying the attention of IT managers in U.S.-based firms. The term "offshore sourcing" includes both offshore outsourcing to a third-party provider as well as offshore insourcing to an internal group within a global corporation [2]. Organizations that avail themselves of outsourcing services can concentrate in their core businesses, potentially reducing the software development team. The combination of these factors results in a significant reduction in the time and cost of software development.

IT managers are being pressured to contain costs in addition to ramp up projects quickly, to find experienced staff in fast-moving technologies, and to innovate constantly. To acquire the IT competencies that address these challenges, managers can choose one of two strategies: either outsource to a domestic supplier or go offshore. The foreign sourcing of IT work is growing based mainly on:

- The increasingly modular design has reduced transactions costs - that is, the cost of coordinating software development and support work between two or more parties. More modular software production eases the burden of synchronizing, communicating, traveling, monitoring, providing feedback, and enforcing contracts;

- Technologies for managing and coordinating work across geographic distances have matured considerably;

- Offshore organizations have improved their software development and project management capabilities.

In the study conducted by Carmel [2], offshore IT sourcing was identified as a maturation process.

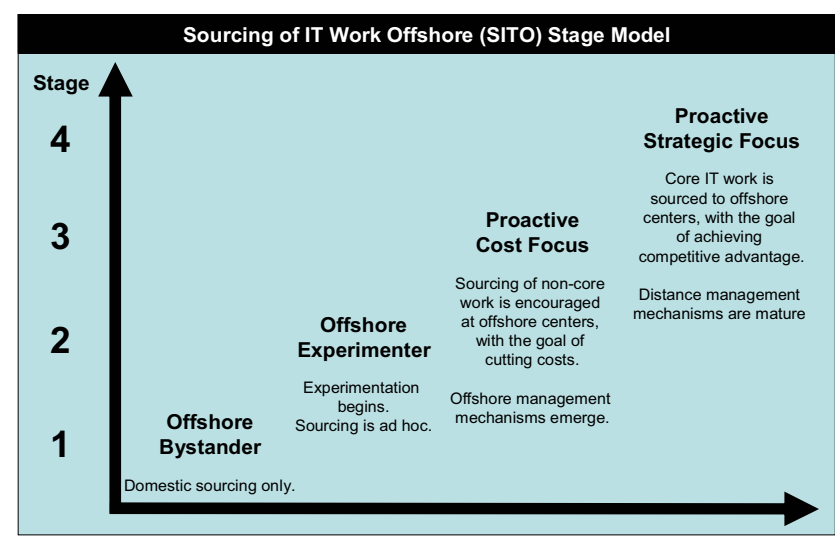

Figure 1. The SITO Model [2]

The 4 dimensions of the stage model presented in Figure 1 are: offshore bystander, offshore experimenter, proactive cost focus, and proactive strategic focus. Each stage is characterized by a set of strategic imperatives and internal firm dynamics. The offshore insourcing is referenced in stage four. Carmel [2] suggests that 
technology companies in stage four have different organizational structures and mechanisms. These firms have accumulated considerably more experience in offshore IT sourcing, but they usually preferred to own their IT units, sourcing from within their firms. The main characteristics of wholly owned offshore units are:

- Sophisticated marketing efforts: since they are not part of the domestically located core IT function, they need an active and aggressive marketing strategy;

- Distinct organizational functions: some of these firms used their offshore IT units for software product R\&D, internal information system work, and providing IT professional services to other firms. Some firms can support all types of software work, while others can support one or two;

- The value of vertical integration: these firms prefer the "build versus buy" argument, and they also prefer having vertical integration and an internal locus of control. Moreover, the ramp-up time is shorter because internal contracting is simpler, insourcing have advantages in the areas of security, confidentiality, and maintaining proprietary knowledge. Finally, internal IT professionals use standard software engineering tools, methodologies, and work process, which reduce the project management problem.

On the other hand, problems do exist and benchmarking with other organizations in similar situations can improve the addressing of these problems, one of the objectives of our program of research.

\subsection{Alternative Offshore Outsourcing Arrangements}

Alternatives to purely contractual relationships do exist: Alliances (e.g., [16], [13]), Joint-ventures (e.g., [6]) and wholly-owned subsidiaries, each with a specific set of pros and cons.

Alliances create a relatively egalitarian connection with the foreign partner but without either partner loosing its identity, whereas a joint-venture presupposes the creation of a new jointly owned entity, many times with its own identity. In both cases, the absence of complete control by the "owning" partner precludes the complete imposition of standards or say, task allocation. We see these alternatives as intermediate points in a control continuum anchored on one side by contracts and on the other by wholly owned subsidiaries. Therefore, our discussion of focusing on the extreme case is likely to bring into stark foreground the differences from the contractually based relationship.

In fact, some of the traditional issues in offshore outsourcing relate to the service provider's responsiveness, compliance, cooperation, and commitment - all in the context of a contractual relationship with a relatively unknown partner. For a more complete discussion, the reader can go to Swinarski et al [15], or Levina and Ross [10].

The issues discussed are related to the relative perceived power between client and service provider; although they can be partly addressed in a contract, ultimately a disagreement in a contract is likely to be ironed out so much later in court that for the purposes of solving an immediate issue it is practically irrelevant.

Moreover, different service providers have been rated on their quality by many sources; however, the more highly rated ones are sometimes more expensive, to the point where the much touted cost advantage can disappear.

On the other hand, the balance of power is completely different in a WOS relationship with the headquarters. Not only the headquarters are the "de facto" boss, but there is also a clear interest on both sides to create and keep a long-term relationship with all its facets.

One of the consequences is that there is no concern or doubt on responsiveness, compliance, cooperation or even commitment on the level existent on traditional offshore outsourcing. But other concerns are still present: not only the time and space separation are still there, but now there is also an explicit "personalization" of the relationships.

The issues of knowledge transfer are now subtly changed as well - the question is not anymore as much about the transfer of sensitive technology, but about the inherent job transfer, with human resource repercussions for the headquarters and subsidiaries. The need for proving one's worth vis-à-vis the other side starts to affect choice of justifying metrics, assignments, and task coordination.

Ultimately, these are the issues that will still have to be addressed when traditional offshore outsourcing investigation solves the problems mentioned above. This study is designed to leapfrog such traditional questions and investigate the latter set of substantive issues.

\subsection{Theoretical Underpinnings}

The initial part of our proposed research program on distributed projects in the WOS realm is the development of a maturity model. In the past, stage models have been the target of much criticism, particularly with regards to their ability of being tested/proved/disproved.

In this section of the manuscript, we will discuss the variables involved in our research. These variables and their measurement is what differentiates our study from the past stage models, because it enables true falsifiability of the model. Second, we will integrate our dimensions and variables into a coherent model by introducing the appropriate theoretical lenses.

\subsubsection{Dimensions of Maturity}

First, we need to have a better understanding of how distributed a project can be. We borrow from Evaristo [4], who suggests dimensions to the concept of 
"distributedness" through a theory-based model. The main objective is to understand what "distributed" means when discussing the management of distributed IT projects and to suggest better ways to manage them by finding out what are the critical problems. These dimensions are presented in Figure 2.

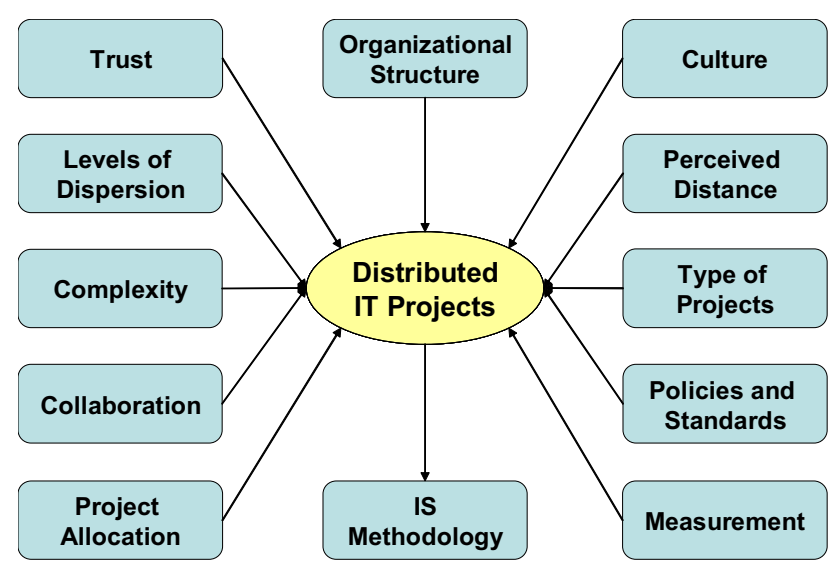

Figure 2. DSD dimensions, adapted from Evaristo [4]

Trust is critical to keep the esprit-de-corp, or "teamness", in a team; however, it is something difficult to create, keep and manage on the day-to-day operation of a DSD team.

In a related fashion, unnoticed cultural differences could magnify problems that may occur because of the inherent difficulty in distributed environments to notice and correct potential misunderstandings. Worst yet, such perception may only develop too late.

Organizational structure includes the type of stakeholders and how headquarters and subsidiaries as well as subsidiary and subsidiary relate to each other. Different types of stakeholders exist in each project, each with different perceptions about the project. The type of projects (development, support, test, etc.), affects the way it should be managed and developed.

The perceived distance is related to the ability of meeting a person face to face frequently (very close) or hardly ever being able to do so (very far). This can be applied to all project team regardless of role.

Collaboration is the extent to which distributed people may be working on the same project concurrently or not. In this case, synchronicity is one of the variables, where all stakeholders are in the same time zone or need to work at the same time on a given project.

The complexity level affects the performance of distributed IT projects. Some examples include the technology used in the project as well as its size. The dimension labeled information systems methodology refers to the type of development methodology used in the project (say, waterfall versus object oriented).

The dimension policies and standards include all standards used in the organization. Among them, programming standards and the choice of applying for software quality certification (say, Capability Maturity Model - CMM).

The level of dispersion can be described as the weighed average of the perceived distance by critical mass of members in each of the locations that comprise a particular project. The higher the dispersion level, the more difficult is to monitor the behavior of different groups and their interaction.

The measurement dimension includes several metrics related to the IS development process, productivity, and cost measurements. Finally, the project allocation dimension is related to how projects are allocated to productive units (subsidiaries).

\subsubsection{Operationalization of the dimensions}

For each dimension, variables will be identified. Some variables of interest, originating from the dimensions described previously include the level of use of coordination tools, knowledge management systems, existence of accepted practices (say, for those companies involved in CMM certification efforts, the level at where each of the wholly owned subsidiary as well as the headquarters are), several metrics related to the quality of brand new products as part of a larger blueprint, maintenance of legacy systems, testing of both old and new systems, completely new systems, and others.

In addition, other variables include user satisfaction with the interaction and results obtained with the wholly owned subsidiary, negotiation and commitment (also called coordination in the sense of completing an agreement within a reasonable time frame), satisfaction of developers in the interaction with their immediate clients, impact of outsourcing on turnover and job satisfaction, and levels of user vs. client involvement.

Finally, variables such as levels of collaboration between offshore subsidiaries, task allocation and distribution model, project life cycle, productivity in the wholly owned subsidiary, resource allocation, project management maturity, decision making, and communication are also important. Table 1 shows some of the identified variables related to its dimension. 
Table 1. Dimensions and some of their variables

\begin{tabular}{|c|c|}
\hline Dimensions & Variables \\
\hline Trust & "Teamness" \\
\hline Organizational Structure & $\begin{array}{l}\text { Type of stakeholders } \\
\text { Number of subsidiaries }\end{array}$ \\
\hline Culture & $\begin{array}{l}\text { National culture } \\
\text { Organizational culture }\end{array}$ \\
\hline Perceived Distance & $\begin{array}{l}\text { Travel } \\
\text { Communication }\end{array}$ \\
\hline Type of Projects & $\begin{array}{l}\text { Development } \\
\text { Support } \\
\text { Enhancement } \\
\text { Test } \\
\text { Preventive maintenance } \\
\text { Corrective maintenance }\end{array}$ \\
\hline Policies and Standards & $\begin{array}{l}\text { Software Quality } \\
\text { Certification }\end{array}$ \\
\hline Measurement & $\begin{array}{l}\text { Productivity } \\
\text { Cost }\end{array}$ \\
\hline Systems Methodology & $\begin{array}{l}\text { Development process } \\
\text { Project life cycle }\end{array}$ \\
\hline Project Allocation & $\begin{array}{l}\text { Decision making } \\
\text { Negotiation } \\
\text { Resource allocation } \\
\text { Distribution model }\end{array}$ \\
\hline Collaboration & $\begin{array}{l}\text { Synchronicity } \\
\text { Tools }\end{array}$ \\
\hline Complexity & Task Allocation \\
\hline Levels of Dispersion & $\begin{array}{l}\text { Subsidiary location } \\
\text { Stakeholder location }\end{array}$ \\
\hline
\end{tabular}

The dependent variable is the level of maturity of the relationship between the headquarters and the subsidiary.

\subsubsection{Theoretical Lenses}

In a macro perspective, the ultimate reason for instituting a wholly-owned subsidiary originates from the make or buy conundrum. Transaction cost theory (TCT) [17] tries to explain this behavior; the main argument is that as long as the cost of making something is smaller than going to market, hierarchies will be created to make a gadget or perform a service. Naturally, the organizational motivation to going to a wholly owned subsidiary under the TCT lens is that is more convenient and in the long run less expensive to make than buy those services from a contractually based relationship. A second perspective is immediately raised: once the subsidiary exists, then another "make-buy" decision occurs when the headquarters are trying to decide whether a particular system should be outsourced to the WOS or not. So TCT is still very relevant. In our model, TCT has the power to explain the relationship between several of the dimensions and the dependent variable.

However, some of the remaining variables can be better explained by agency theory (AT) [7]. For instance, the dimension of "culture" presupposes expectations of certain behaviors for an agent that may or may not be aligned with the interests of the principal. Similarly, when the perceived distance is large, monitoring costs increase, affecting the agency costs. In this particular case, TCT would also come in to help explain the eventual decision of make or buy.

\subsubsection{Conclusion}

DSD dramatically impacts the way products are conceptualized, developed, tested and delivered. Therefore, the structure needed to support such environment is also completely different, both in processes as well as in technology. The introduction of a strong theoretical lens based on transaction cost theory and agency theory strengthens our model and enables the development of theoretically based expectations of how certain variables will affect our dependent variable. Details not before obvious grow in importance; this research will uncover some of these issues as well.

\section{Research Method}

Historically, many of the stage models proposed (i.e. Nolan's [12]) have been criticized because of the inherent difficulty in testing the different stages. Therefore, most of the models, including the Capability Maturity Model (CMM) is based on a description of behaviors that when observed show that a particular organization seems to be in a particular phase or stage.

The first phase of this research is an exploratory study with the objective of increasing knowledge where typically practice has preceded theory. The main research method in this phase will be the case study [18]. Figure 3 presents the main phases in the study.

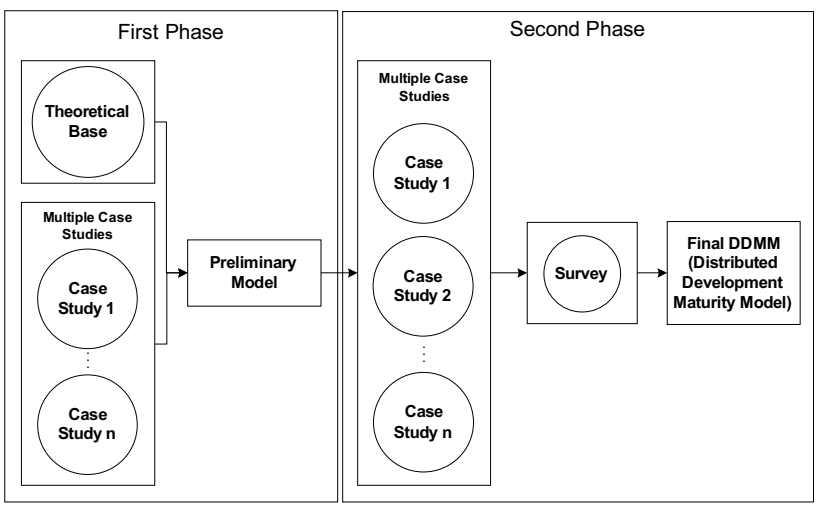

Figure 3. The research plan

The objective of the initial phase is to propose a preliminary maturity model of software development in offshore insourcing based not only on the extensive combined experience of the researchers in this area but also on a theoretical and empirical approach. Concurrently with a complete literature review, several case studies will be performed using software projects as the unit of analysis. 
These sets of case studies will be conducted (according to Yin [18], and Kripendorff [9]) using several data collection methods, allowing for triangulation of data and therefore increasing the result reliability and quality. In particular, we will use interviews, semi-structured and cross-sectional surveys, and document analysis. Basically, questions relating to the variables proposed earlier will be prepared with two objectives: first, to arrive at a qualitative understanding of the phenomenon, its causes and effects; second, to use that understanding to inform future choices of case studies and to develop an efficient and parsimonious subset of relevant variables and their measurement for the second phase of the study, detailed below.

The second phase will be two-pronged: (a) carefully chosen case studies based on needs identified in the first phase and (b) a survey. The convenience sample will include large organizations that are heavily involved in IT and which have at least one wholly owned subsidiary centered on IT development. The condition for participation is commit to not only a certain number of hours for the initial interviews by key informants, but also individuals' time along the quantitative data collection to enable survey responses at different points in time.

The case studies will validate and extend the preliminary model generating an improved preliminary model to be fully validated by the survey, with the objective of mapping its characteristics on a global level. The survey will be based on a parsimonious set of variables resulting for the first phase and will be made available to participating organizations through the web. Several waves of data collection are planned. Benefits for those organizations participating in the study include benchmarking. The survey will enable the measurement of all independent variables as well as the operationalization for the dependent variable. As a result, the last step is then to propose a Maturity Model of IT Distributed Projects in Offshore Insourcing Environments (DDMM).

A third phase (not represented in Figure 3) longitudinal or on-going - will include the organizations' participation for continued data collection efforts using the same instrument every 6 months to monitor changes in the environment. It is expected that one of the results of this quantitative phase will be a set of values for the independent variables that will characterize a particular stage.

\section{Research Objectives and Expected Results}

The objective of this research program is to understand the dynamics of management of the wholly owned subsidiaries for IT development. In order to do this, we propose a maturity model for distributed IT projects for those organizations involved in offshore insourcing environments. To achieve this objective, we define the following specific goals:
- Learning from and improving the theory on offshore sourcing and DSD;

- To identify the main characteristics of the offshore insourcing environment;

- To develop a preliminary model that identifies the main DSD environments and their relationship with the offshore insourcing environment;

To identify other aspects related to all stakeholders involved in the offshore insourcing.

\subsection{Expected Results}

At the end of the research, we expected the following results:

- The maturity model for distributed IT projects in offshore insourcing environments;

- A description of the different maturity phases, with a hard specification of what characterizes each phase in terms of the key variables of interest;

- A "future view" of which issues to expect as one moves forward, and solutions that have worked for other organizations;

- The ability to benchmark the organizations against others;

- Development of a body of knowledge on "best practices";

- Development of action items that allow your organization to move faster to the more appropriate stage based on your needs and reality;

- Develop technical reports and papers to validate the model in the industry and academic communities.

\section{Conclusion}

According to Morstead [11], offshore IT services have the potential to save organizations $35 \%-65 \%$ on their inhouse costs. It is estimated that in India alone, the size of the market will grow from $\$ 10.3$ billion in 2001 to $\$ 77$ billion in 2008. But in a world where domestic initiatives often suffer from poor planning and cost overruns, the stories of offshore failures can be alarming.

Moving an onshore process offshore is not a simple step; it's not even a simple project. Is hard work, but it is a real and achievable goal. But the strategy carries unique obstacles. Communications, policies, methods, culture, and process details are just one facet of the unique characteristics of offshore environments. New problems and obstacles emerge and many scenarios are possible, including the offshore insourcing (or wholly owned subsidiaries).

The wholly owned subsidiary offers the advantages of full control, since it is completely subordinate to the parent corporation. This can be attractive to either organizations that make a strategy decision of moving part of their development offshore at the policy level or those that may hope for cost reduction and higher control in the future, than it would be otherwise possible in traditional offshore outsourcing. 
This project is not only a landmark study in the area of offshore insourcing (or wholly-owned subsidiaries), something until recently not been researched, but also has strong implications to the more traditional offshore outsourcing. The key reason is that most of the work currently being done in offshore outsourcing is seen under the perspective of contracting; although obviously very relevant, eventually such studies will need to go further past that issue - which is exactly what we are proposing

\section{References}

[1]E. Carmel, Global SoftwareTeams: Collaborating Across Borders and Time Zones, Upper Saddle River, New Jersey: Prentice Hall PTR, 1999.

[2]E. Carmel and R. Agarwal, "The Maturation of Offshore Sourcing of Information Technology Work," MIS Quarterly Executive, vol. 1, no. 2, 2002, pp. 65-77.

[3]D. Damian, "The Study of Requirements Engineering in Global Software Development: As Challenging as Important," in Proceedings of Proceedings of the International Workshop on Global Software Development at ICSE, Florida, 2002.

[4]J.R. Evaristo, R. Scudder, K. Desouza, and O. Sato, "A Dimensional Analysis of Geographically Distributed Project Teams: A Case Study," Journal of Engineering and Technology Management, vol. forthcoming, 2004.

[5]J. Herbsleb, A. Mockus, T. Finholt, and R. Grinter, "An Empirical Study of Global Software Development: Distance and Speed," in Proceedings of 23rd International Conference on Software Engineering, 2001.

[6]A. Inkpen and A. Dinur, "Knowledge Management Processes in International Joint Ventures," Organization Science, vol. 9, no. 4, 1998, pp. 454-468.

[7]M.C. Jensen and W.H. Meckling, "Theory of the Firm: Managerial Structure, Agency Costs, and Ownership Structure," in J. Barney and W. Ouchi, ed., Organizational Economics, San Francisco: Jossey Bass Publishers, 1986, pp. 214-275.
[8]D.W. Karolak, "Managing Virtual Teams and Environments," IEEE Computer Society, 1998.

[9]K. Krippendorf, Content Analysis: An Introduction to its Methodology, Sage, 1980.

[10]N. Levina and J. Ross, "From the Vendor's Perspective: Exploring the Value Proposition in Information Technology Outsourcing," MIS Quarterly, vol. 27, no. 3, 2003, pp. 331-364.

[11]S. Morstead and G. Blount, Offshore Ready, 1st ed. ed., ISANI Press, 2003.

[12]R. Nolan, "Managing the Crisis in Data Processing," Harvard Business Review, vol. 57, no. 2, 1979, pp. 115126.

[13]J.-G. Oh, "Global Strategic Alliances in the Telecommunications Industry," Telecommunications Policy, vol. 20, no. 9, 1996, pp. 713-720.

[14]R. Prikladnicki, J. Audy, and J.R. Evaristo, "Distributed Software Development: Toward an understanding of the Relationship between Project Team, Users, and Customers," in Proceedings of ICEIS, Angers, France, 2003.

[15]M. Swinarski, R. Kishore, and H.R. Rao, "The Effects of Power and Partnership on Application Service Provider Commitment, Cooperation, and Compliance," in Proceedings of 37th Hawaii International Conference on Systems Sciences, Big Island of Hawaii, Hawaii, 2004.

[16]L. Wildeman, "Alliances and networks: The next generation," International Journal of Technology

Management, vol. 15, no. 1,2, 1998, pp. 96-108.

[17]O. Williamson, Markets and Hierarchies, New York: Free Press, 1975.

[18]R. Yin, Case Study Research, Design and Methods, 2nd ed., Applied Social Research Methods Series, Vol. 5, Thousand Oaks, California: Sage Publications, Inc., 1994.

\footnotetext{
${ }^{\mathrm{i}}$ Research partially developed at CDPe research center at PUCRS University, and funded in terms of the Brazilian Federal Law for Information Technology (Law No. 8.248/91).
} 